\title{
Fidelity and concurrence of entangled qudits between a trapped ion and two laser beams
}

\author{
Rasim Dermez $^{a, c, *}$, S. Abdel-Khalek ${ }^{b}$, and E. M. Khalil ${ }^{b}$ \\ ${ }^{a}$ Department of Physics, Afyon Kocatepe University, Afyonkarahisar, 03200, Turkey. \\ *e-mail:dermez@aku.edu.tr \\ ${ }^{b}$ Department of Mathematics, College of Science, Taif University, Taif, Saudi Arabia \\ (sabotalb@tu.edu.sa). \\ ${ }^{c}$ Vocational School of Afyon, Afyon Kocatepe, University, Afyonkarahisar, 03200, Turkey.
}

Received 9 March 2021; accepted 20 April 2021

\begin{abstract}
A quantum scheme is presented in which a three-level trapped ion interacts with two laser beams in the absence and presence of a classical field. We analyze the impact of the classical field and the Lamb-Dicke parameter (LDP) on the dynamical behavior of an entanglement quantifier, population probabilities and the geometric phase (GP). From the set of LDP values and the classical field, we obtain the time dependence of the GP and population probabilities. The results show that both the time-dependent field and the LDP play an important role in developing the entanglement, fidelity and population probabilities. Robust entangled qudit states are obtained by two elaborated measures which are concurrence and fidelity.
\end{abstract}

Keywords: Lamb-Dicke parameter; trapped three-level ion; geometric phase; fidelity; concurrence.

PACS: $03.65 . \mathrm{Ud} ; 74.25 . \mathrm{Kc} ; 65.40 . \mathrm{Gr}$.

DOI: https://doi.org/10.31349/RevMexFis.67.050705

\section{Introduction}

Studies on entangled states have increased over the past years owing to further development of quantum mechanics [1-5]. Although Einstein and Schrödinger discovered entanglement in 1935 [6,7], some of its properties remain as a mystery. Schrödinger proposed his famous thought experiment where a cat is in a superposition the two distinct states "alive" and "dead". The life of the cat is uncertain until a measurement determines its destiny. Entanglement is the special state of two Schrödinger cats in quantum superposition. In the same year, Niels Bohr published an article [8] with the same title as the Einstein-Podolsky-Rosen article. Entanglement is a property of correlations between two or more quantum systems. These correlations defy classical description and are associated with intrinsically quantum phenomena. Researchers have made considerable contributions to quantum communication that have produced several approaches to allocating bipartite entanglement [4-9]. Entanglement of qutrit states [9] is attested by a quantum structure for the lower order terms of the density matrix. It is characterized by quantum dynamics of a three-level trapped ion using the quantum entropy of the system with a time-dependent Rabi frequency [10]. This entanglement is addressed with quantum correlations in negativity $(N)$ and concurrence $(C)[9,10]$ concerning the reduced and the total density matrices. Quantum discord is an optimal source for the difference between total correlation and classical correlation in a bipartite system $[7,11]$.

In nonclassical state generation in trapped ions, an external classical driving field is used to manipulate the coupling of internal electronic levels of the ion with its phonons. Phys- ical models of such ion-phonon systems contain exponential nonlinearities in the motional degrees of freedom. However, these properties of entanglement result from the assumed linearity in the Lamb-Dicke limit (LDL). Beyond the LDL, the coupling between the internal and the external degrees of freedom becomes nonlinear. Studying the persistence of such effects beyond the LDL requires going beyond the linear assumption. A further rotating-wave approximation (RWA) yields the two-level quantum optical Hamiltonian governing phonon dynamics.

The GP has drawn considerable attention as a worthwhile means of attaining different phenomena in the processing of fault-tolerance in quantum information. In this sense, many scientists investigated some applications of this phenomenon [12-14]. First, we can review the outcomes as a modulation during the stage introduced by Berry [15]. It is shown that the Berry's phase might be modified by weakly dissipative "Liouville equation for the density matrix" through presenting imaginary correction [16] or damping the density matrix elements [17]. Recently, the influence of time-dependent coupling on the evolution of GP via the atomic speed and acceleration has been examined [18]. It is shown that the dynamic behavior of the GP and the entanglement of a threelevel atom with two fields in presence of the RWA include a richer structure than the absence of the RWA [19]. AbdelKhalek et al. [20] studied the impact of the cavity damping on the features of the GP and the entanglement of the three-level atom and field.

Trapped ions represent the most interesting applications of quantum bits (qubits) in processing quantum information because of long coherence times [21]. Ions restricted to a lin- 
ear radio-frequency (Paul) trap are cooled with a spatial array. The Hilbert space of the composite quantum system takes the form

$$
C^{d}=C^{d_{\text {ion }}} \otimes C^{d_{p}}
$$

where $d_{\text {ion }}=3$ and $d_{\text {phonon }}=4$ are the dimensions of the three-level ion and photons, respectively. It is shown that the quantum dynamics with $C$ [22], $N$ [23], and quantum entropy [10] for a trapped three-level ion that interacts with two photons in time-dependent Hamiltonians. $N$ and $C$ are two measure of entanglement, which is a valuable representation in quantum information, especially in the ionic system. Entangled base and product base can be presented as a generalization of Schmidt coefficients. [24] show that the vibronic motion of a trapped ion far from the Lamb-Dicke regime (LDR) can be defined. It is shown that the full information on the quantum state of the vibrational center-of-mass (c.m.) motion of a trapped ion can be transferred to its electronic dynamics by long-living electronic transition by laser light [25].

A configuration for generating even and odd coherent states of the motion of a trapped ion is proposed with respect to laser excitation of two vibronic transitions [26]. Qubits are robust against noise $a_{1}|0\rangle+a_{2}|1\rangle$ (such as right panel of Eq. (6) can be generated with arbitrary amplitudes [27]. An approximate equivalence between a generalized quantum Rabi model and its nth order counterparts is shown [28]. The general character of nonlinear coherent states has been idendified [29]. These experiments were performed beyond the LDR with an assumed value of $\eta$, including $\eta \sim 0.2$ [30]. Quantum entanglement of the Fock states is investigated by a quadratic Hamiltonian [31]. A qubit can be cereated in the interaction of the displaced Fock states of the electromagnetic field with a two-level atom in RWA [32].

The outline of this paper is as follows. Section 2 presents the theoretical model and its solution under a classical field. The evolution of a qubit pure state from an initially unentangled state is described in Sec. 3. Section 4 investigates the temporal behavior of the GP and the population probabilities. Section 5 quantifies the entanglement according to the concurrence and the fidelity of the proposed system. Our main findings are summarized in Sec. 6.

\section{Theoretical model and its solution under the classical field}

A trapped three-level ion interacting with two laser beams in $\Lambda$ configuration is proposed. In this quantum system, the dimension of Hilbert space is 12 . The quantum dynamics of the trapped ion-phonon system has been previously investigated [8-10,33]. Additionally, the status of an ion-phonon system is also valid for superposition of macroscopic quantum states with coupling of two laser beams [34]. Trapped ions are considered one of the best candidates to perform quantum information. Many of the Ion-laser interactions cover the complete solutions [35]. The probability amplitudes of atomic levels of ion are given: $|e\rangle=|1\rangle$ trapped ion excited level, $|r\rangle=|2\rangle$ raman level, and $|g\rangle=|3\rangle$ ground level are taken as $1 / \sqrt{2}$. Thus, a beam may lead a transition only. The $\Lambda$ configuration can be equal to a cascade $\Xi$ scheme for two laser beams.

The $\Lambda$ scheme, namely a quantum scheme, in our model system is characterized by the total Hamiltonian $\hat{H}=\hat{H}_{\text {ion }}+$ $\hat{H}_{1}+\hat{H}_{2}+\hat{H}_{\mathrm{c}}$. The trapped ion Hamiltonian $\hat{H}_{\text {ion }}$ and interaction Hamiltonians $H_{1}$ and $H_{2}$ are given as $(\hbar=1)$

$$
\begin{aligned}
\hat{H}_{\mathrm{ion}} & =\frac{p_{x}^{2}}{2 m}+\frac{1}{2} m \nu^{2} \hat{x}+\omega_{e}|e\rangle\left\langle e\left|+\omega_{r}\right| r\right\rangle\left\langle r\left|+\omega_{g}\right| g\right\rangle\langle g| \\
\hat{H}_{1} & =\frac{i \Omega}{2} \mathrm{e}^{i\left(k_{1} \hat{x}-\omega t\right)}(|e\rangle\langle g|-| g\rangle\langle e|) \\
\hat{H}_{2} & =\frac{i \Omega}{2} \mathrm{e}^{i\left(-k_{2} \hat{x}-\omega t\right)}(|e\rangle\langle r|-| r\rangle\langle e|) \\
\hat{H}_{c} & =\varepsilon\left(\hat{S}_{r g}+\hat{S}_{g r}\right)
\end{aligned}
$$

where mass of trapped ion is $m$, the harmonic trap frequency

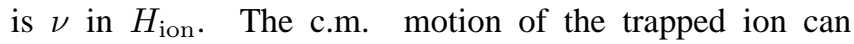
be described using the standard harmonic oscillator quantization of $H_{\text {ion. }}$. This quantization includes the momentum and position operators $p_{x}=i \sqrt{(m \nu / 2)}\left(a^{\dagger}-a\right)$ and $x=\sqrt{(1 / 2 m \nu)}\left(a+a^{\dagger}\right) . a$ and $a^{\dagger}$ are the annihilation and creation operators of the phonons. The trapped ion Hamiltonian is similar to the quantum Rabi interaction Hamiltonian has similarities to $H_{\text {ion }}$ [36]. The wave numbers $k_{1}$ and $k_{2}$ characterize the two laser beams.

The matrix $\hat{S}_{r g}$ is the r-g interaction of the classical field [37], and $\hat{S}_{g r}$ the vice versa interaction. The last term $\hat{H}_{c}$ represents the classical field, which has coefficient $\varepsilon$. Equation (2) includes the raman level energy $\omega_{r}$, the ground level energy $\omega_{g}$ and the excited level energy $\omega_{e}$. The frequencies of the two laser beams are supposed to be equal $\omega=\omega_{1}=\omega_{2}$ and the Rabi frequencies of the dipole interactions between the lasers and the trapped ion are given by $\Omega$. Thus, equality of Rabi frequencies such as $\Omega=\Omega_{1}=\Omega_{2}$ is given by the interaction Hamiltonians, in $H_{1}$ and $H_{2}$.

The relation between the Rabi and the harmonic trap frequencies is in the form of $\Omega / 2=\nu$ in ion-phonon system. The transition Rabi frequencies $\Omega_{e-g}$ and $\Omega_{e-r}$ are assumed to be equal and represented by

$$
\left(\begin{array}{l}
|e\rangle \\
|r\rangle \\
|g\rangle
\end{array}\right)=\left(\begin{array}{ccc}
1 & 0 & 0 \\
0 & \cos \beta & \sin \beta \\
0 & -\sin \beta & \cos \beta
\end{array}\right)\left(\begin{array}{l}
|1\rangle \\
|2\rangle \\
|3\rangle
\end{array}\right)
$$

with $\tan (2 \beta)=\arctan \left(2 \varepsilon / \omega_{g}-\omega_{r}\right)$. Here, $\beta$ is the classical field effect. $\tilde{H}=U^{\dagger} \hat{H} U$ is transformed Hamiltonian. By means of this Hamiltonian, the mathematical hierarchy from Eq. (2) to Eq. (4) is briefly: The total Hamiltonian, Eq. (2) considered in the study is the energy of the ion-phonon system. In Schrödinger Picture and unitary transform, using the Planck constant equal to one, $\hbar=1$, this Hamiltonian, Eq. 
(4) has been obtained by the transmission process. Transformation matrix $U$ is written as [34],

$$
U=\frac{1}{2}\left(\begin{array}{ccc}
0 & \sqrt{2} & \sqrt{2} \\
-\sqrt{2} D[\eta] & D[\eta] & -D[\eta] \\
\sqrt{2} D[-\eta] & D[-\eta] & -D[-\eta]
\end{array}\right) .
$$

Glauber displacement operators are given as $D( \pm \eta)=$ $e^{ \pm \mathrm{i} \eta\left(a+a^{\dagger}\right)}$. When we use the above transformation and apply the RWA, the system of the transformed Hamiltonian is found to be $\tilde{H}=\tilde{H}_{0}+\tilde{V}$, where

$$
\begin{aligned}
\tilde{H}_{0} & =\nu \hat{a}^{\dagger} \hat{a}+\omega_{e}|1\rangle\left\langle 1\left|+\Omega_{r}\right| 2\right\rangle\left\langle 2\left|+\Omega_{g}\right| 3\right\rangle\langle 3| \\
\tilde{V} & =\frac{i \mu_{1}}{2} \mathrm{e}^{i\left(k_{1} \hat{x}-\omega t\right)}(|1\rangle\langle 2|-| 2\rangle\langle 1|) \\
& +\frac{i \mu_{2}}{2} \mathrm{e}^{i\left(k_{2} \hat{x}-\omega t\right)}(|1\rangle\langle 3|-| 3\rangle\langle 1|)
\end{aligned}
$$

here

$$
\begin{aligned}
& \Omega_{r}=\omega_{r} \cos ^{2} \beta+\omega_{\beta} \sin ^{2} \beta-\beta \sin (2 \beta) \\
& \Omega_{g}=\omega_{g} \cos ^{2} \beta+\omega_{r} \sin ^{2} \beta+\beta \sin (2 \beta) \\
& \mu_{1}=\Omega(\cos \beta-\sin \beta) \\
& \mu_{2}=\Omega(\sin \beta+\cos \beta) .
\end{aligned}
$$

Two studies on ion-laser interaction help us understand photon dynamics $[35,38]$. The Hamiltonian of our model system is

$$
\begin{aligned}
H & =\nu a^{\dagger} a-\delta|1\rangle\left\langle 1\left|+\frac{\Omega}{2} e^{i \eta\left(a^{\dagger}+a\right)}\right| 1\right\rangle\langle 3| \\
& +\frac{\Omega}{2} e^{-i \eta\left(a^{\dagger}+a\right)}|1\rangle\langle 2|+\text { h.c. }
\end{aligned}
$$

The transformation is performed with the matrix $U_{0}=$ $\exp (-i \omega t|e\rangle\langle e|)$ [34]. We consider two lasers, of wavelength $\lambda$ that are somewhat red detuned from the upper level $\mid e>$ by the same amount $\delta=-\nu \eta^{2}$, where $\eta$ is the LD parameter. The lasers are characterized by $\eta=k / \sqrt{2 m \nu}$, with $k=2 \pi / \lambda$. In the weak excitation regime with $\Omega=2 \nu$, the lead $\Lambda$ scheme is equivalent to a cascade $\Xi$ based on a unitary transformation [34] which is characterized by $U$. We introduce the basis vectors $\langle 1|=\langle e|=(1,0,0),\langle 2|=\langle r|=$ $(0,1,0)$ and $\langle 3|=\langle g|=(0,0,1)$, so the transformed Hamiltonian $\tilde{H}=U^{\dagger} \hat{H} U$ takes the form

$$
\begin{aligned}
\tilde{H} & =\nu a^{\dagger} a+\nu \eta^{2}+\nu(|2\rangle\langle 2|-| 3\rangle\langle 3|) \\
& -i \mu\left(a^{\dagger}|1\rangle\left\langle 2\left|-a^{\dagger}\right| 3\right\rangle\langle 1|+\text { h.c. }\right),
\end{aligned}
$$

where, $\mu=\nu \eta / \sqrt{2}$. Both phonon transitions are resonant and have the same strength. This Hamiltonian is attained with an RWA that is valid for $\eta<1 / \alpha$, where $\alpha$ is the amplitude of coherent displacements of the phonons [34]. Small coherent displacements $(\alpha \ll 1)$ are studied, so that the range of $\eta$ is from 0.01 to 0.1 . From the unitary transformation [34], the time evolution of an original state $\psi(0)$ is defined

$$
|\psi(t)\rangle=U_{0}^{\dagger} U e^{-i t \tilde{H}_{0}} K(t) U^{\dagger}|\psi(0)\rangle .
$$

where $K(t)$ represents the eminent propagator for the cascade Hamiltonian [34], and $e^{-i t \tilde{H}_{0}}$ is the rotating frame transformation representing the interaction. This shows the original state of our system, where the lower-order subsystem is in a coherent state. Under such settings, we obtain a robust entangled state in Figs. 1-4.

\section{Evolution of a qubit pure state from an ini- tially unentangled state}

In the initial state of the ion-phonons system, the phonon subsystem is in a coherent state and the trapped ion is in the superposition state of its ground and raman levels that is $|\psi(0)\rangle=(1 / \sqrt{2})(|g\rangle-|r\rangle) \otimes \sum_{n=0}^{\infty} F_{n}|n\rangle$. The generalized coherent state is $F_{n}(\alpha)=e^{-|\alpha|^{2}} / 2\left(\alpha^{n} / \sqrt{n !}\right)$ in this equation. $n=0$ and $n=1, F_{0}=1$ and $F_{1}=\alpha$ are the amplitudes of the Fock number states of two phonons. The amplitude of the ground state is $1 / \sqrt{2}$, the raman state is $-1 / \sqrt{2}$ and the excited state is zero. We suppose a minor vibration of an ion in a harmonic trap and coherent state $|\alpha\rangle$ is described with $\alpha \ll 1$. We have taken $a=1$ and $\alpha=0.05$ for two coherent states in Eq.(6). The two phonons normalization condition is $\|a\|^{2}+\|\alpha\|^{2}=|1|^{2}+|0.05|^{2} \simeq 1$. The first order in $\alpha$ is the case of approximately normalized phonons. The normalization condition for two amplitudes of the trapped ion is exactly $[1 / \sqrt{2}]^{2}+[-1 / \sqrt{2}]^{2}=1$. Therefore, the state of the trapped ion-phonon system is

$$
|\psi(0)\rangle=(1 / \sqrt{2})(|3\rangle-|2\rangle) \otimes(a|0\rangle+\alpha|1\rangle .
$$

In optimal conditions, only a small mean photon number is required for producing entanglement.

This composite state is transformed into an initial case of the cascade $|\tilde{\psi}(0)\rangle=U^{\dagger}|\psi(0)\rangle=\sum_{\sigma, n} A_{\sigma, n}|\sigma, n\rangle$, where $\sigma=1,2,3$ represents atomic levels, and $n=0,1,2,3$ represents vibrational numbers. First-order corrections yield $\sum_{\sigma, n} A_{\sigma, n}(t)|\sigma, n\rangle$, the coefficients of the system's probability amplitudes are

$$
\begin{aligned}
& A_{10}(t)=\left[\cos (\sqrt{1 / 2} t)+\frac{i \eta}{\sqrt{2}} \sin (\sqrt{1 / 2} t)\right] \exp [-i t / \eta] \\
& A_{11}(t)=\alpha \cos (\sqrt{3 / 2} t) \exp [-i t / \eta] \\
& A_{12}(t)=-\frac{i \eta}{\sqrt{5}} \sin (\sqrt{5 / 2} t) \exp [-2 i t / \eta] \\
& B_{20}(t)=\frac{\alpha}{\sqrt{3}} \sin (\sqrt{3 / 2} t) \exp [-i t / \eta] \\
& B_{21}(t)=\frac{i \eta}{\sqrt{2}}\left[\frac{3}{5}+\frac{2}{5} \cos (\sqrt{5 / 2} t)\right] \exp [-2 i t / \eta] \\
& C_{31}(t)=\left[\sin (\sqrt{1 / 2} t)-\frac{i \eta}{\sqrt{2}} \cos (\sqrt{1 / 2} t)\right] \exp [-i t \eta] \\
& C_{32}(t)=\alpha \sqrt{\frac{2}{3}} \sin (\sqrt{3 / 2} t) \exp [-i t / \eta]
\end{aligned}
$$




$$
C_{33}(t)=-\frac{\sqrt{3}}{5} i \eta(1-\cos (\sqrt{5 / 2} t)) \exp [-2 i t / \eta],
$$

where $t$ is dimensionless time scaled with $\nu \eta$ (harmonic trap frequency-the LDP). In Figs. 1-4, a scaled time of 5 is equal to 500 microseconds for $\mathrm{LDP}=0.01$. The calculation is as follows: If $\eta=0,01$ and $\nu=1 \times 10^{6} \mathrm{~Hz}$, then $\nu \eta=10^{4}$, $(5 / \nu \eta)=500 \times 10^{-6}=500$ microseconds. With the back unitary transformation $U$ from the $\Xi$ scheme to the $\Lambda$ scheme, the wave function in the original space $(e, r, g)$ takes the form

$$
\begin{aligned}
|\psi(t)\rangle & =\sum_{n=0}^{3}\left(u_{n}(t)|e, n\rangle+\left[v_{n}(t) \cos \beta-w_{n}(t) \sin \beta\right]|r, n\rangle\right. \\
& \left.+\left[v_{n}(t) \sin \beta+w_{n}(t) \cos \beta\right]|g, n\rangle\right)
\end{aligned}
$$

According to Eq. (3) and Eq. (4), the state vector of the system is precise in the infinite-dimensional Hilbert space. The situation of the system [34] is much more complicated than our system in the limited Hilbert space. The measurement of entanglement is very hard to comprehend owing to the complex construction of the system and the infinite-dimensional Hilbert space. The exact system is unclear in this case. Thus, we limit the Hilbert space so that the quantum state can be easily examined through Eq. (8). This equation eliminates the difficulties encountered in quantum measurements. The noteworthy coefficients becomes;

$$
\begin{aligned}
& u_{i}(t)=\frac{1}{\sqrt{2}} e^{-i \omega t / \nu \eta}\left[A_{2 i}(t)+A_{3 i}(t)\right], \quad(i=0,1,2,3), \\
& v_{0}(t)=-\frac{1}{\sqrt{2}} A_{10}(t)+\frac{1}{2} A_{20}(t)-\frac{i \eta}{2} A_{31}(t), \\
& v_{1}(t)=-\frac{i \eta}{\sqrt{2}} A_{10}(t)-\frac{1}{\sqrt{2}} A_{11}(t)+\frac{1}{2} A_{21}(t)-\frac{1}{2} A_{31}(t), \\
& v_{2}(t)=-\frac{1}{\sqrt{2}} A_{12}(t)-\frac{i \eta}{\sqrt{2}} A_{31}(t)-\frac{1}{2} A_{32}(t), \\
& v_{3}(t)=-\frac{1}{2} A_{33}(t)=w_{3}(t), \\
& w_{0}(t)=\frac{1}{\sqrt{2}} A_{10}(t)+\frac{1}{2} A_{20}(t)+\frac{i \eta}{2} A_{31}(t), \\
& w_{1}(t)=-\frac{i \eta}{\sqrt{2}} A_{10}(t)+\frac{1}{\sqrt{2}} A_{11}(t)+\frac{1}{2} A_{21}(t)-\frac{1}{2} A_{31}(t), \\
& w_{2}(t)=\frac{1}{\sqrt{2}} A_{12}(t)+\frac{i \eta}{\sqrt{2}} A_{31}(t)-\frac{1}{2} A_{32}(t),
\end{aligned}
$$

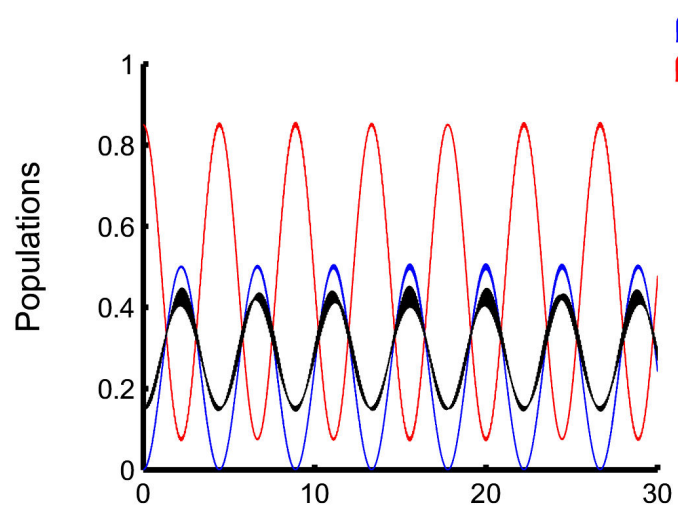

a)

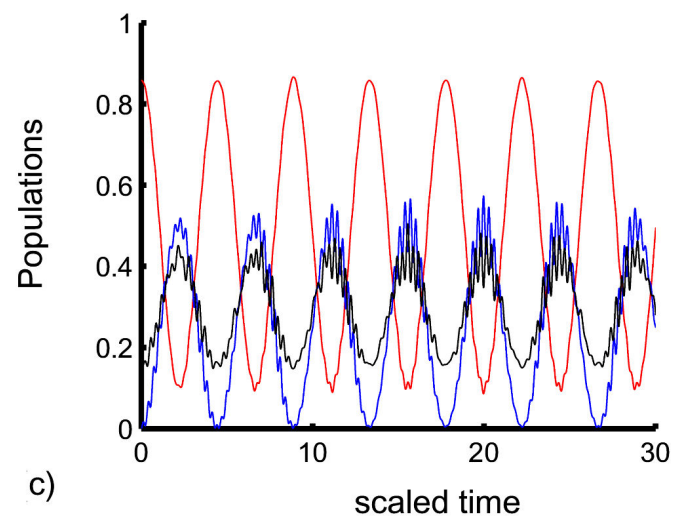

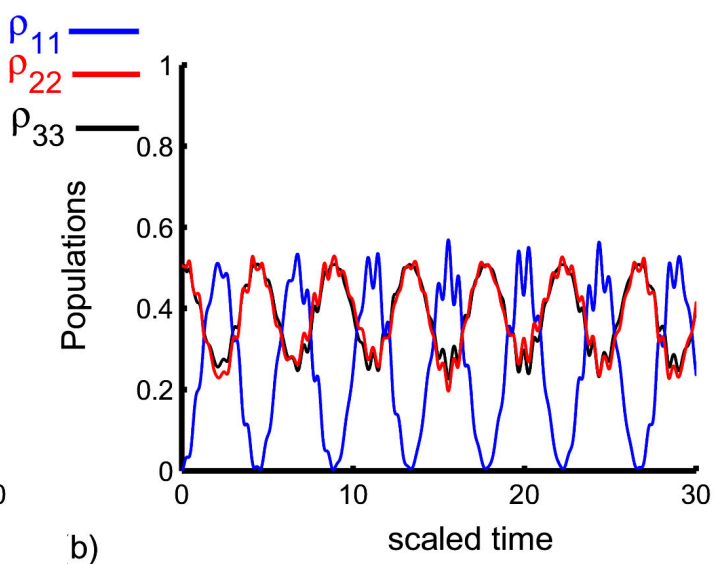

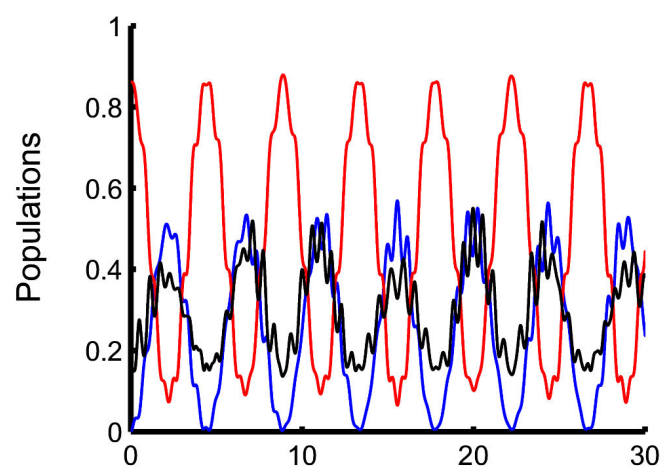

d)

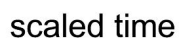

FIGURE 1. Population probabilities $\rho_{11}$ (blue curve), $\rho_{22}$ (red curve), and $\rho_{33}$ (black curve) versus scaled time for $\alpha=0.05$ with the two LDPs $\eta=0.01$ (a,c) and $\eta=0.1$ (b,d) using the Planck constant equal to one, $\hbar=1$. The classical field is ignored (i.e. $\beta=0$ ) in $(\mathrm{a}, \mathrm{c})$ and considered for $\beta=0.49$ in $(\mathrm{b}, \mathrm{d})$. The variable $t$ is dimensionless time scaled with $\nu \eta$. The other coupling parameters are $\omega_{e g}=5 \times 10^{14} \mathrm{~Hz}$ and $\nu=1 \mathrm{MHz}$. 
where $\omega=\omega_{e g}-\eta^{2} \nu$ and $\omega_{e g}=\omega_{13}=5 \times 10^{14} \mathrm{~Hz}$ is the resonance frequency of the $g-e$ transition.

\section{Theoretical framework of population prob- abilities and the GP}

The atomic population is very important in studying the entanglement between the parts of the system. In this section, we study the effects of both the classical field and the LDP on the atomic population.

First, we neglect the classical field $(\beta=0)$ and take the smallest value of the LDP $(\eta=0.01)$. It is clear that $\rho_{11}(t)$ oscillates periodically between 0 and 0.5 , while both $\rho_{22}(t)$ and $\rho_{33}(t)$ fluctuate identically between 0.25 and 0.5 as shown in Fig. 1a). The maximum values of the function $\rho_{22}(t)$ increases strongly and reaches 0.83 , but the amplitude of the oscillations decreases with respect to $\rho_{33}(t)$ after the classical field is added $(\beta=0.49)$. The quantity $\rho_{11}(t)$ is not affected as expected (the classical field rotates between $\rho_{22}(t)$ and $\left.\rho_{33}(t)\right)$, as shown in Fig. 1(b). The fluctuations increase with the parameter $\eta$. The atomic population has the same behavior as in the previous case with an increasing maximum value under the classical field, as evident in Figs. 1c) and 1d). Here $\rho_{11}$ is the first diagonal term $|e\rangle\langle e|$ for the reduced density operator in Eq. (13). Similarly, other two terms are $\rho_{22}=|r\rangle\langle r|$ and $\rho_{33}=|g\rangle\langle g|$. For the noncyclic evolution, the TI-laser field may be defined by a final wave function that is diverse and not obtained from the initial wave function. This function proliferates with a complex factor according to

$$
\langle\psi(0) \mid \psi(t)\rangle=\kappa \exp (i \Lambda)
$$

where $\kappa$ indicates a real scalar. GP is the phase achieved over the evolution of a state from $|\psi(0)\rangle$ to $|\psi(t)\rangle$ according to $[39,40]$

$$
\Phi_{g}=\arg \{\langle\psi(0) \mid \psi(t)\rangle\}
$$

The classical field is initially excluded, and the LDP is at its smallest value $(\eta=0.01)$. The GP oscillates harmonically between $-\pi$ and $\pi$ with a period of $3 \pi$, as shown in Fig. 2a). These oscillations have rectangular form in the middle of the period, precisely at $(3 \pi / 2)$. The rectangular oscillations in
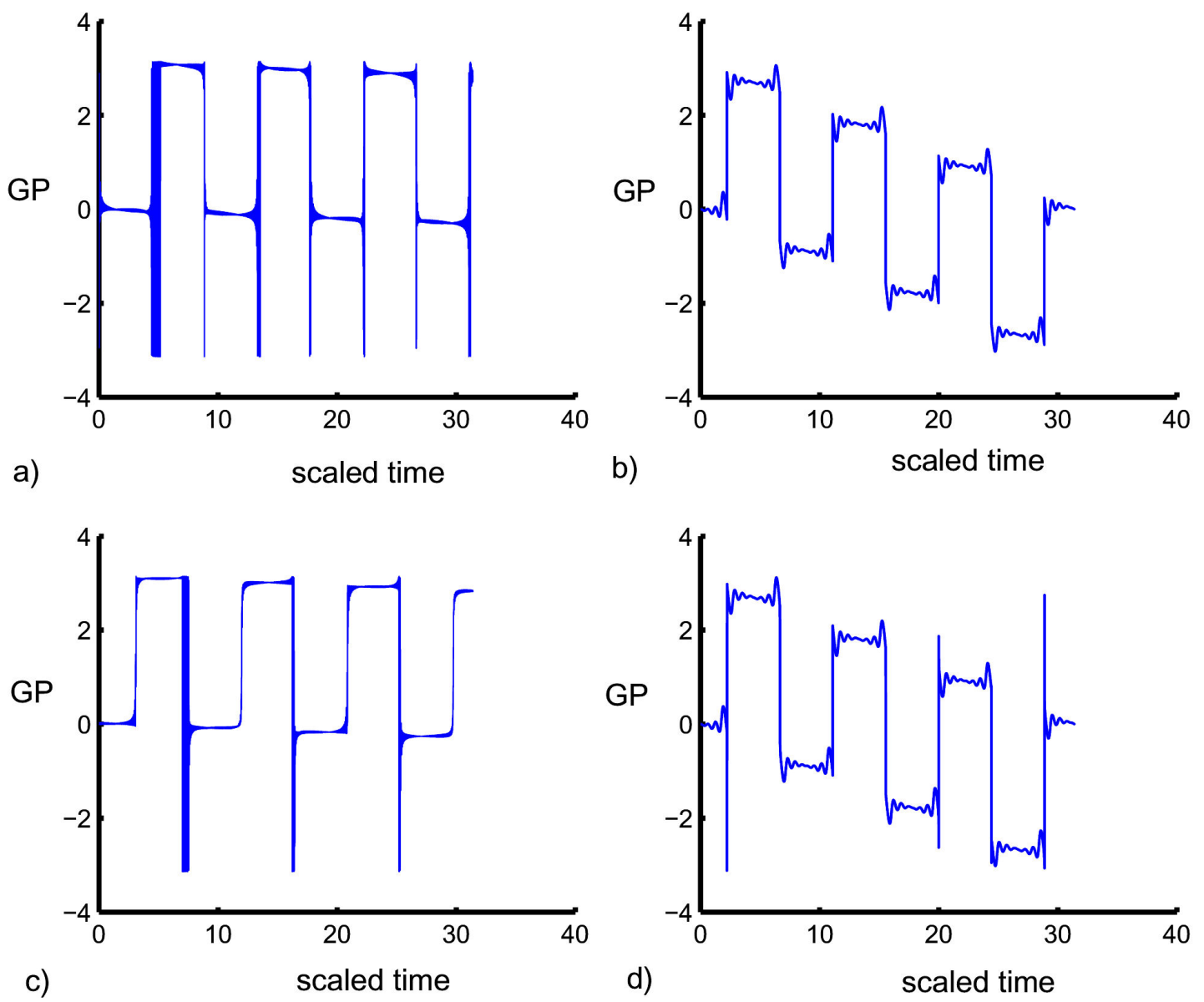

FIGURE 2. GP versus scaled time for $\alpha=0.05$ with the two LDPs $\eta=0.01$ (a,c) and $\eta=0.1$ (b,d) using the Planck constant equal to one, $\hbar=1$. The classical field is ignored (i.e. $\beta=0)$ in $(\mathrm{a}, \mathrm{c})$ and considered for $\beta=0.49$ in $(\mathrm{b}, \mathrm{d})$. The other coupling parameters are the same as Fig. 1. 

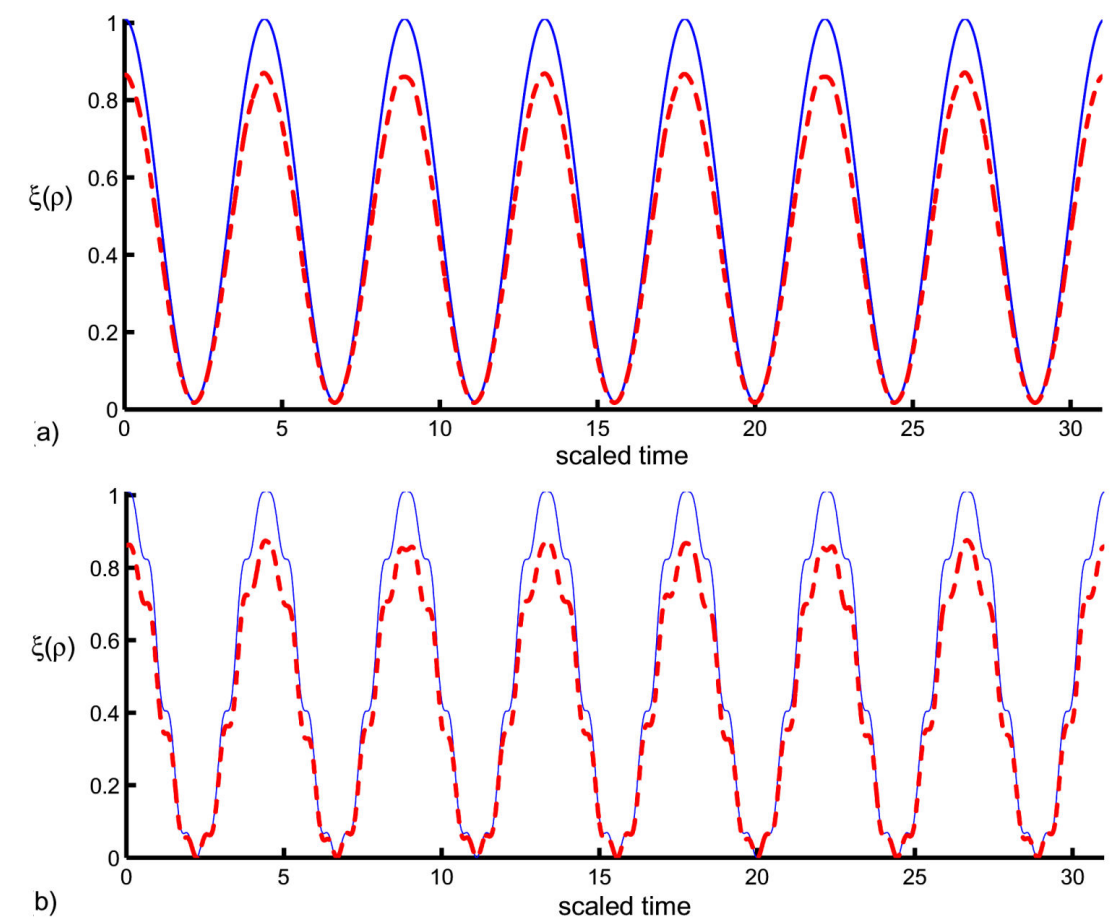

FIGURE 3. Fidelity $\xi(\rho)$ versus scaled time for $\alpha=0.05$. The classical field is ignored (i.e. $\beta=0$ ) along the (blue curve) and considered for $\beta=0.49$ along the (dashed red curve). The two LDP values are $\eta=0.01 \mathrm{a}$ ) and $\eta=0.1 \mathrm{~b}$ ). The other coupling parameters are the same as Fig. 1.

the previous case are shifted to the end of the period, precisely at $(3 \pi)$, after adding the classical field is added in to the interaction cavity $(\beta=0.49)$. The negative values of the GP function are reduced, as seen in Fig. 2b). Considering the large value of the LDP $(\eta=0.1)$ and the exclusion of the classical field, the GP has more oscillations and the fluctuations are still vertical between $-\pi$ and $\pi$, as shown in Fig. 2c). The oscillations are reduced after the classical field is taken into account, as shown in Fig. 2d).

\section{Entanglement measures and fidelity}

In the quantum information scheme, fidelity is used to evaluate the transformation between the changing and fixed states. In this part, we study fidelity defined as $[41,42]$

$$
\xi(t)=\sqrt{\langle\psi(0)|\hat{\rho}(t)| \psi(0)\rangle}=|\langle\psi(0) \mid \psi(t)\rangle| .
$$

where $|\psi(t)\rangle$ is given by Eq. (7).

For the minimum LDP value $(\eta=0.01)$, the fidelity fluctuates smoothly between 0 and 1 every $\pi$ without the classical field. This indicates weak entanglement between the two phonons and the trapped ion. The function $\xi(t)$ decreases after the classical field is added to the interaction cavity, as shown in Fig. 3a). Previous oscillations are decreased slightly after the LDP is increased $(\eta=0.01 \rightarrow 0.1)$, so the parameter is influenced by the entanglement of the system parts. Comparing the previous results, we notice that the classic field reduces the entanglement in ion-phonon system, as shown in Fig. 3b).
The total density operator $\rho_{\text {ion }- \text { phonon }}$ is represented by a $12 \times 12 \mathrm{D}$ matrix in the base of $|\mathrm{i}, p\rangle$. When the trace is taken over the phonon system, the $3 \times 3$ reduced density operator is

$$
\begin{aligned}
\rho_{\text {ion }} & =\operatorname{Tr}_{\text {phonon }}\left(\rho_{\text {ion }- \text { phonon }}\right) \\
& =\left(\begin{array}{lll}
\operatorname{Tr}|e\rangle\langle e| & \operatorname{Tr}|e\rangle\langle r| & \operatorname{Tr}|e\rangle\langle g| \\
\operatorname{Tr}|r\rangle\langle e| & \operatorname{Tr}|r\rangle\langle r| & \operatorname{Tr}|r\rangle\langle g| \\
\operatorname{Tr}|g\rangle\langle e| & \operatorname{Tr}|g\rangle\langle r| & \operatorname{Tr}|g\rangle\langle g|
\end{array}\right),
\end{aligned}
$$

where the diagonal terms, $|e\rangle\langle e|| r\rangle,\langle r|$, and $|g\rangle\langle g|$ are a $3 \times 3$ matrix. Taking the trace over the phonon system reduces from the $12 \times 12$ matrix to a $3 \times 3$ matrix. The total density operator of the system takes the form

$$
\rho_{\text {ion-phonon }}=(|\psi(t)\rangle\langle\psi(t)|),
$$

where $|\psi(t)\rangle\langle\psi(t)|$ denotes a $12 \times 12$ matrix in the Hilbert 12 -space. We get the following analytic solution of the total density operator

$$
\rho_{\text {ion-phonon }}=U^{\dagger}(t)\left[\rho^{\mathrm{i}}(0) \otimes \rho^{\mathrm{p}}(0)\right] U(t) .
$$

An arbitrary bipartite pure state is written as [43-45]

$$
C(t)=\sqrt{2\left(\operatorname{Tr}\left(\rho_{\mathrm{i}-p}\right)-\operatorname{Tr}\left(\rho_{\mathrm{ion}}^{2}\right)\right)} .
$$

where $\rho_{\text {ion }}=T r_{\text {phonon }}\left(\rho_{\text {ion-phonon }}\right)$ is the reduced density operator. We are currently discussing concurrence through 

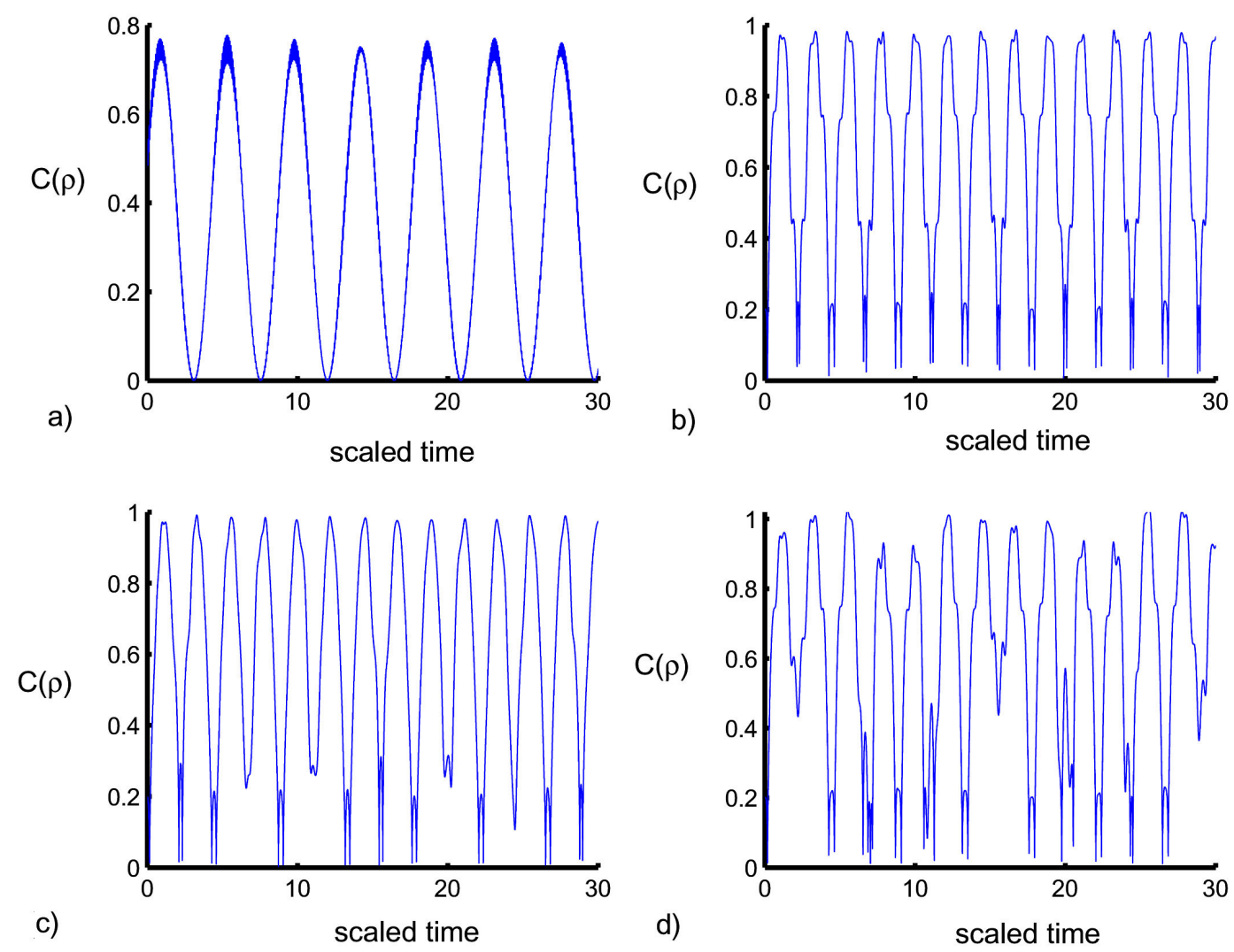

FIgURE 4. Concurrence versus scaled time for $\alpha=0.05$, with LDPs $\eta=0.01$ (a,c) and $\eta=0.1$ (b,d). The classical field is ignored (i.e. $\beta=0)$ in $(\mathrm{a}, \mathrm{b})$ and considered for $\beta=0.49$ in $(\mathrm{c}, \mathrm{d})$. The other coupling parameters are the same as Fig. 1.

the same aforementioned conditions. For the smallest values of the LDP $(\eta=0.01)$ without the classical field, the concurrence $C(t)$ oscillates regularly between 0 and 1 and reaches the minimum values periodically, as shown in Fig. 4a). The oscillations of the function $C(t)$ increase and decrease to the smallest values after the increase in $\eta$.

In addition, the maximum values are raised to the top by increasing $\eta$ as shown in Fig. 4c). For the small values of the $\eta$, the high values of the function $C(t)$ rise sharply. Furthermore, some lower values decrease after the addition of the classical field to the interaction cavity as see in Fig. 4c). While the maximum values that is the peaks decrease, the minimum increases after the parameter $\eta$ increases. Therefore, entanglement is greatly influenced by the parameter $\eta$ of the classical field as shown in Fig. 4d).

Consequently, the optimum values in our figures agree with the results of other studies, that deal with elaborated quantum measurements such as fidelity and $C$ [7, 46-51]. This is because, in an ion-phonon system, the amount and duration of entanglement are developed in comparison with other studies.

\section{Conclusion}

We considered the interaction between a three-level trapped ion with two laser beams in a $\Lambda$ (lambda) configuration. The general solution for the present system was obtained with a wave function. Using this wave function in 12-D space, we obtained an analytic solution of the total density operator, $\rho_{\text {ion-phonon }}=U^{\dagger}(t)\left[\rho^{\mathrm{i}}(0) \otimes \rho^{\mathrm{p}}(0)\right] U(t)$. The resulting population and GP were considered in the presence and absence of a classical field. It turns out that the classical field strongly affects the population and the geometric phase. We used four different variations of these two factors, the LDP and the classical field. We observed that the physical and dynamical properties of the quantum quantifiers can be controlled by appropriately selecting the classical field and the LDP. In population inversion, the amplitude of the oscillations decreases in the presence of the classical field. The oscillations improve as the LDP increases. In the GP, the negative portions decrease after the classical field is included in the interaction cavity.

When the classical field comes from 0 to 0.49 , fidelity and $C$ values rise in Figs. 3-4. We can say that fidelity and $C$ are two measures elaborated for entangled qudit states. The phenomenon of sudden death and sudden birth decreases with increasing LDP and the classical field. The entanglement fluctuates regularly with increasing LDP and reaches a state of separation periodically. This insight may have useful applications in quantum optics and information processing. 


\section{Acknowledgments}

Taif University Researchers Supporting Project number (TURSP-2020/17), Taif University, Taif, Saudi Arabia. Besides, this work was supported by Afyon Kocatepe Univer- sity 09-FENED.06 in Turkey. M. Kurban from Edanz Group (https://en-author-services.edanz.com/ac) edited a draft of this manuscript. We thank S. Tellioglu and B. Avsar for their scientific contributions.
1. R. Dermez, Generalized Concurrence and Negativity in TimeDependent $C^{3} \times C^{5}=C^{15}$ Dimensional Ionic-Phononic Systems, J. Russ. Laser Res. 37 (2016) 572, https://doi. org/10.1007/s10946-016-9609-1

2. G. Gour and B C Senders, Remote Preparation and Distribution of Bipartite Entangled States, 93 (2004) 572, https://link.aps.org/doi/10.1103/ PhysRevLett.93.260501

3. A. G. Rodriguez, Entangled states and the singular value decomposition, Rev. Mex. Fis. 46 (2000) 419.

4. S. Ahadpour and F. Mirmasoudi, The role of noisy channels in quantum teleportation, Rev. Mex. Fis. 66 (2020) 378, https : //doi.org/10.31349/RevMexFis.66.378

5. R. Dermez and Ö. E. Müstecaplıŏlu, Long-lived entangled qudits in a trapped three-level ion beyond Lamb-Dicke limit, Phys. Scr. 71 (2009) 015304, https://doi.org/10.1088/ 0031-8949/79/01/015304

6. E. Schrödinger, Die gegenwärtige Situation in der Quantenmechanik, Naturwissenschaften 23 (1935) 807 https:// doi.org/10.1007/BF01491891

7. A. Einstein, B. Podolsky, N. Rosen, Can quantum-mechanical description of physical reality be considered complete?, Phys. Rev. 47 (1935) 777, https://link.aps.org/doi/10. 1103/PhysRev.47.777

8. N. Bohr, Can quantum-mechanical description of physical reality be considered complete?, Phys. Rev. 48 (1935) 696, https://doi.org/10.1103/PhysRev.48.696

9. R. Dermez and S. Özen, Higher dimensional entangled qudits in a trapped three-level ion, Eur. Phys. J. D. 57 (2010) 431, https://doi.org/10.1140/epjd/ e2010-00051-6

10. R. Dermez, B. Deveci and D. Ö. Güney, Quantum dynamics of a three-level trapped ion under a time-dependent interaction with laser beams, Eur. Phys. J. D 67 (2013) 120, https : //doi.org/10.1140/epjd/e2013-30649-9

11. M. Ramzan, Decoherence dynamics of discord for multipartite quantum systems, Eur. Phys. J. D 67 (2013) 170, https: //doi.org/10.1140/epjd/e2013-30700-y

12. M. Abdel-Aty, A qualitative perspective on the dynamics of a single-Cooper-pair box with a phase-damped cavity, J. Phys. A: Math. Theor. 41, (2008) 185304, https://doi.org/ 10.1088/1751-8113/41/18/185304.

13. S. Abdel-Khalek, K. Berrada, H. Eleuch, M. Abel-Aty, Dynamics of Wehrl entropy of a degenerate two-photon process with a nonlinear medium, Opt. Quant. Electron. 42 (2011) 887, https://doi.org/10.1007/s11082-011-9498-z
14. T. M. El-Shahat, S. Abdel-Khalek, A.-S. F. Obada, Entropy squeezing of a driven two-level atom in a cavity with injected squeezed vacuum, Chaos, Solitons \& Fractals 26 (2005) 1293, https://doi.org/10.1016/j.chaos. 2005.03 .013

15. M. V. Berry, Quantal Phase Factors Accompanying Adiabatic Changes, Proc. R. Soc. A 392 (1984) 45.

16. D. Ellinas, S. M. Barnett and M. A. Dupertuis, Berry's phase in optical resonance, Phys. Rev. A 39 (1989) 3228, https : //doi.org/10.1103/PhysRevA.39.3228.

17. D. Gamliel and J. H. Freed, Berry's geometrical phases in ESR in the presence of a stochastic process, Phys. Rev. A 39 (1989) 3238, https://doi.org/10.1103/PhysRevA. 39.3238

18. S. Abdel-Khalek, Quantum Entanglement and Geometric Phase of Two Moving Two-Level Atoms, Open Systems and Information Dynamics 22 (2015) 1550015, https://doi. org/10.1142/S1230161215500158

19. S. Abdel-Khalek, Y. S. El-Saman, I. Mechai, M. AbdelAty, Geometric Phase and Entanglement of a Three-Level Atom With and Without Rotating Wave Approximation, Braz. J. Phys. 48 (2018) 9, https://doi.org/10.1007/ s13538-017-0537-5

20. S. Abdel-Khalek, Y.S. El-Saman, I. Mechai, M. AbdelAty, Some features of the geometric phase and entanglement of three-level atom under cavity damping effects, Indian J. Phys. (2019) 1698, https://doi.org/10.1007/ s12648-019-01613-5

21. P. Maunz, S. Moehring, K. C. Younge, D. N. Matsukevich, C. Monreo, Quantum interference of photon pairs from two remote trapped atomic ions, Nature Phys. 3 (2007) 538, https: //doi.org/10.1038/nphys644

22. A. S. F. Obada, S. Abdel-Khalek and D. A. M. Abo-Kahla, New features of entanglement and other applications of a two-qubit system, Optics Commun. 283 (2010) 4662, https: / / doi. org/10.1016/j.optcom.2010.06.074

23. J. S. Kim, A. Das and B. C. Sanders, Entanglement monogamy of multipartite higher-dimensional quantum systems using convex-roof extended negativity, Phys. Rev. A. 79 (2009) 012329, https://doi.org/10.1103/PhysRevA.79. 012329 .

24. W. Vogel and R. L. de Matos Filho, Nonlinear JaynesCummings dynamics of a trapped ion, Phys. Rev. A 52 (1995) 4214, https: //doi.org/10.1103/PhysRevA. 52.4214

25. S. Wallentowitz and W. Vogel, Reconstruction of the Quantum Mechanical State of a Trapped Ion, Phys. Rev. Lett. 75, (1995) 2932, https://doi.org/10.1103/ PhysRevLett.75.2932 
26. R. L. de Matos Filho and W. Vogel, Even and Odd Coherent States of the Motion of a Trapped Ion, Phys. Rev. Lett. 76 (1996) 608, https://doi.org/10.1103/ PhysRevLett. 76.608

27. H. Moya-Cessa and P. Tombesi, Filtering number states of the vibrational motion of an ion, Phys. Rev. A 61 (2000) 025401, https://doi.org/10.1103/PhysRevA.61. 025401 .

28. J. Casanova, R. Puebla, H. Moya-Cessa and M. B. Plenio, Connecting nth order generalised quantum Rabi models: Emergence of nonlinear spin-boson coupling via spin rotations, npj Quantum Inf. 4 (2018) 47, https: / / doi.org/10.1038/ s41534-018-0096-9

29. Z. Kis, W. Vogel, and L. Davidovich, Nonlinear coherent states of trapped-atom motion, Phys. Rev. A 64 (2001) 033401, https://doi.org/10.1103/PhysRevA.64. 033401 .

30. C. Monreo, D. M. Meekhof, B. E. King, D. J. Wineland, A "Schrödinger Cat" Superposition State of an Atom, Science 272 (1996) 1131, 10.1126/science.272.5265.1131.

31. S. Hacyan, Evolution and entanglement of Fock states, Rev. Mex. Fis. 43, (1996) 519.

32. L.M. A. Aguilar and H. Moya-Cessa, Quantum bits and superposition of displaced Fock states of the cavity field, Rev. Mex. Fis. 48,(2002) 423.

33. S. Lee, D.P. Chi, S. D. Oh and J. Kim, Convex-roof extended negativity as an entanglement measure for bipartite quantum systems, Phys. Rev. A 68 (2003) 062304, https://doi. org/10.1103/PhysRevA.68.062304

34. Ö. E. Müstecaplığlu, Motional macroscopic quantum superposition states of a trapped three-level ion, Phys. Rev. A 68, (2003) 023811, https://doi.org/10.1103/ PhysRevA.68.023811

35. H. Moya-Cessa, F. Soto-Eguibara, J. M. Vargas-MartÃnez, R. Juarez-Amaro and A. Zuniga-Segundoc, Ion-laser interactions: The most complete solution, Physics Reports 513 (2012) 229, https://doi.org/10.1016/j. physrep.2012.01.002

36. I. Ramos Prieto and H.M. Moya-Cessa, Eigenestados exactos y estados entrelazados de un ion atrapado oscilando en dos dimensiones, Rev. Mex. Fis. 63, (2017) 194.

37. M.S. Abdalla, A.-S. F. Obada, E.M. Khalil, Atom-field interaction under the influence of two external classical and quantum fields, Optics Comm. 285 (2012) 1283, https : / / doi . org/ $10.1016 / j . o p t c o m .2011 .10 .038$

38. H. Moya-Cessa, D. Jonathan and P. L. Knight, A family of exact eigenstates for a single trapped ion interacting with a laser field, Journal of Modern Optics 50 (2003) 265, https : //doi.org/10.1080/09500340308235175

39. S. Pancharatnam, Generalized theory of interference, and its applications, Proc. Indian Acad. Sci. 44 (1956) 247, https : //doi.org/10.1007/BF03046050
40. M. Abdel-Aty, S. Abdel-Khalek and A. S.-F. Obada, Pancharatnam Phase of Two-Mode Optical Fields with Kerr Nonlinearity, Optical Review 7 (2000) 499.

41. J.-X. Wang, X.-J. Zhang and X.-X. Zhang, Evolution Properties of Atomic Fidelity in the Combined Multi-Atom-Cavity Field System, Commun. Theor. Phys. 63 (2015) 749, https : //doi.org/10.1088/0253-6102/63/6/749

42. S. V. Prants, M. Yu. Uleysky and V. Yu. Argonov, Entanglement, fidelity, and quantum-classical correlations with an atom moving in a quantized cavity field, Phys. Rev. A 73 (2006) 023807, https://doi.org/10.1103/PhysRevA. 73. 023807

43. W. K. Wootters, Entanglement of Formation of an Arbitrary State of Two Qubits, Phys. Rev. Lett. 80 (1998) 2245, https: //doi.org/10.1103/PhysRevLett.80.2245.

44. S. Hill and W. K. Wootters, Entanglement of a Pair of Quantum Bits, Phys. Rev. Lett. 78 (1997) 5022, https: //doi.org/ $10.1103 /$ PhysRevLett. 78.5022

45. L. Hughston, R. Jozsa and W. K. Wootters, A complete classification of quantum ensembles having a given density matrix, Phys. Lett. A 183 (1993) 14, https://doi.org/10. 1016/0375-9601(93) 90880-9

46. M. Abdel-Aty, Information entropy of a time-dependent threelevel trapped ion interacting with a laser field, J. Phys. A. Math. and Gen. 38 (2005) 8589, https: //doi.org/10.1088/ $0305-4470 / 38 / 40 / 008$

47. M. Abdel-Aty, Influence of a Kerr-like medium on the evolution of field entropy and entanglement in a three-level atom, J. Phys. B: At. Mol. Opt. Phys. 33, (2000) 2665, https: //doi.org/10.1088/0953-4075/33/14/305

48. R. Dermez, S. A. Khalek, K. Kara, B. Deveci, and G. N. Gunaydin, Full-trapped three-level ion in the Lamb-Dicke limit: analyzing and comparing quantum entanglement measures of two qudits, J. Russ. Laser Res. 33 (2012) 42, https : / / doi . org10.1007/s10946-012-9257-z

49. R. Dermez, Quantifying of quantum entanglement in Schrödinger cat states with the trapped ion-coherent system for the deep Lamb-Dick regime, Indian J. Phys. 95 (2021) 219, https://doi.org/10.1007/ s12648-020-01697-4

50. R. Dermez, Quantification of Mixed-State Entanglement in a Quantum System Interacting with Two Time-Dependent Lasers, J. Russ. Laser Res. 34, (2013) 192, https://doi.org/ $10.1007 / \mathrm{s} 10946-013-9342-y$.

51. R. Dermez, Comparing concurrence and negativity in timedependent ionic-phononic system with fifteen dimensional density matrix, IOP Publishing Journal of Physics: Conference Series 766 (2016) 012012, https : / / doi .org/10.1088/ $1742-6596 / 766 / 1 / 012012$ 